\title{
High-osmolarity signalling in Saccharomyces cerevisiae is modulated in a carbon-source- dependent fashion
}

\author{
Marco Siderius, Eveline Rots and Willem H. Mager
}

Department of Biochemistry and

Molecular Biology, IMBW, BioCentrum Amsterdam,

Vrije Universiteit,

De Boelelaan 1083,

1081 HV Amsterdam,

The Netherlands
Author for correspondence: Willem H. Mager. Tel: +3120444 7569. Fax: +31204447553. e-mail: Mager@chem.vu.nl

\begin{abstract}
High-osmolarity-induced expression of the small heat-shock gene HSP12 is regulated by the HOG (high-osmolarity glycerol) pathway and PKA (protein kinase A). To analyse the regulatory input of both signal transduction pathways, high-salt-induced HSP12 expression in different genetic backgrounds on glucose-, ethanol- and glycerol-based culture media was examined. Upon exposure to high-osmolarity stress, the kinetics of induction of HSP12 in cells growing on the non-fermentable carbon sources are strikingly different from those on glucose. Derepression of HSP12 gene expression under non-stress conditions was observed in cells growing on non-fermentable carbon sources. High-salt challenge resulted in a lower induction of the HSP12 mRNA levels in ethanol-grown cells as compared to glucose-grown cells, whereas in glycerol-grown cells hardly any high-salt induction of HSP12 MRNA levels could be detected. Analysis of signalling through the HOG pathway suggested that glycerol may influence the activity of this signalling route, possibly via negative feedback. Furthermore, the cellular level of PKA activity was found to have a great impact on stress-responsive gene transcription. On the basis of the data obtained it was concluded that modulation of PKA activity plays a major role in the stress response. A glucose-dependent, PKAregulated cellular component is postulated to affect high-osmolarity-induced HSP12 expression.
\end{abstract}

Keywords: stress response, signal transduction, protein kinase A (PKA), highosmolarity glycerol (HOG) pathway, HSP12

\section{INTRODUCTION}

Being a unicellular organism, Saccharomyces cerevisiae is subject to an ever-changing environment which may challenge its growth potential. To survive, yeast cells must cope with alterations in e.g. temperature, osmolarity, redox conditions and availability of nutrients. To overcome these stress conditions, a set of cellular responses is triggered leading to the immediate repair of cellular damage and adaptation to the encountered situation. Adapted cells are not only able to resume growth, but are also more protected when exposed to a new stress situation (Trollmo et al., 1988; Varela et al., 1992). This protection is not only limited to a second challenge of the same kind of stress, but also to other

Abbreviations: HOG, high-osmolarity glycerol; MAP kinase, mitogenactivated protein kinase; PKA, protein kinase A; STRE, general stressresponsive element. types of stress, indicating that the stress response consists of both specific and general elements (Coote et al., 1991; Trollmo et al., 1988; Varela et al., 1992). Insight into the molecular mechanisms that enable yeast to sense and respond to adverse growth conditions has increased enormously over the last few years with the characterization of stress-responsive genes and signal transduction pathways mediating their expression (for an extensive review, see Hohmann \& Mager, 1997).

The responses of yeast to an increase in the osmolarity of the medium comprise both activation of specific and general stress-induced signal transduction pathways. The high-osmolarity glycerol (HOG) mitogen-activated protein (MAP) kinase pathway was characterized as one of five MAP kinase pathways in yeast (Brewster et al., 1993; Levin \& Errede, 1995). Increasing the osmolarity of the medium triggers the activation of the pathway via two putative high-osmolarity receptors, $\operatorname{Sln} 1 \mathrm{p}$ and Sho1p (Maeda et al., 1994, 1995). Sln1p is the sensor of 
a three-component histidine kinase system with Ypd1p and Ssk1p as response regulators (Posas et al., 1996). Activation by high osmolarity releases a block upon the two MAPKK kinases Ssk2 and Ssk22 after which Pbs2p (MAPK kinase) can stimulate the Hog1p kinase by a characteristic dual phosphorylation. Interestingly, the activity of $\mathrm{Pbs} 2 \mathrm{p}$ can also be regulated directly by Sho1p. The Sho1p induction of Pbs $2 p$ activity is thought to involve interaction of the proteins via an SH3-like domain (Maeda et al., 1995). The activation of Hog1p is a prerequisite for the increase in internal glycerol concentration to compensate for the loss of water during high-osmolarity stress (Brewster et al., 1993). Expression of the gene encoding the key enzyme in glycerol production, GPD1, is controlled by the activity of the HOG pathway (Albertyn et al., 1994; Larsson et al., 1993). However, the mechanism linking Hog1p activation to the downstream responses is unknown.

The small heat-shock protein gene HSP12 is also expressed upon high-osmolarity challenge (Varela et al., 1995). This gene belongs to a vast group of so-called general stress-responsive genes. Like GPD1, high-osmolarity-induced expression of HSP12 is regulated by the activation of Hog1p. Although the function of $\mathrm{Hsp} 12$ in yeast is still unknown, it seems likely that it contributes to the stress adaptation in general, because it is also expressed after heat shock, oxidative stress and under nutrient-limiting conditions (Varela et al., 1995). A second input of signal transduction into the regulation of HSP12 expression is mediated by PKA. HSP12 mRNA levels are increased when PKA activity is lowered, as has been shown by examining PKA-mutant yeast strains.

Deletion analysis of the promoter of HSP12 (Varela $e t$ al., 1995) and of other genes induced by multiple stresses such as CTT1 (Marchler et al., 1993) and DDR2 (Kobayashi \& McEntee, 1993), revealed a general stressresponsive cis-acting element (STRE) to be involved in mediating the general stress signal. STRE sequences are present in the promoter of many stress-response genes in variable amounts and orientation. (Ruis \& Schüller, 1995; Siderius \& Mager, 1997). Whether this reflects a way to fine-tune stress-induced expression is not known. Two proteins, Msn2p and Msn4p, containing zincfinger DNA-binding domains which bind to STRE sequences were shown to mediate the general stressresponse signal (Martinez-Pastor et al., 1996; Schmitt \& McEntee, 1996).

As described in Siderius \& Mager (1997), the only signal which is known to influence the response to a variety of stress conditions in yeast is related to PKA activity. The Ras/PKA pathway has been shown to regulate both growth-related metabolism, e.g. the upshift of ribosomal protein gene expression when shifting to glucose as a carbon source (Griffioen et al., 1994), as well as expression of stress-responsive genes (Marchler et al., 1993; Varela et al., 1995). For this reason PKA activity might be considered as a switch mechanism that yeast cells can use to stop growth and initiate the stress response when necessary. The way PKA activity is regulated is poorly understood. Activation of PKA involves the release of the catalytic subunits $T p k 1,2$ and $3 p$ from their regulatory subunit $B c y 1 p$, as occurs after an increase in the cAMP level in the cell. On the other hand, PKA activity is supposed to be regulated via a cAMP-independent fashion through the fermentablegrowth-medium (FGM) pathway (Thevelein, 1994). It has previously been demonstrated that general stressresponsive transcription is under negative regulation by cAMP. Whether the underlying mechanism resides in the modulation of the affinity of Bcy1p for the catalytic subunits or of their activity is elusive.

To investigate the role of the specific and general signal transduction pathways in the response of yeast to highosmolarity stress, we analysed the kinetics of HSP12 expression in various signalling mutants. To further elucidate the role of PKA activity in stress-induced signal transduction we have also examined the highosmolarity response in yeast cells grown on media with glucose, ethanol or glycerol as sole carbon source. We show that the net output of stress-induced signal transduction varies greatly depending on the carbon source utilized.

\section{METHODS}

Strains and growth conditions. Yeast strains used in this study are listed in Table 1. Yeasts were cultured in YP medium supplemented with glucose $(2 \%, w / v)$, ethanol $(1 \%, v / v)$ or glycerol $(3 \%, v / v)$ or YNB supplemented with the same carbon sources as well as the appropriate amino acids. Standard culturing conditions were at $30^{\circ} \mathrm{C}$ on a rotary shaker (220 r.p.m.) unless indicated otherwise. HSP12 promoter-GUS fusions ( $\Delta 2$ and $\Delta 32$, containing 561 and 234 bp of the $5^{\prime}$ flanking region of the HSP12 start codon, respectively) were inserted in the URA3 locus in the different mutants used in this study and in their isogenic wild-type. Proper integration and copy number were determined by Southern blotting. For the Hog1p phosphorylation analysis, the EG123 strain was transformed with a pMA3a plasmid carrying the HOG1 gene (pFJ0) (Varela et al., 1995).

High-osmolarity-stress experiments and RNA isolation. Cells cultured overnight were diluted to an $\mathrm{OD}_{660}$ of $0 \cdot 1$ (Beckman, DU-6z spectrophotometer) and grown until the earlyexponential phase $\left(\mathrm{OD}_{660}\right.$ of $\left.0 \cdot 2\right)$ and then subjected to highosmolarity stress $(0.7 \mathrm{M} \mathrm{NaCl})$ by addition of a concentrated (5 M) salt solution. Samples were taken at the time-points indicated in the figures by quickly freezing the cells in liquid nitrogen. After thawing the samples in cold water, cells were washed in $1 \mathrm{ml} \mathrm{H}_{2} \mathrm{O}$ and resuspended in $400 \mu \mathrm{l}$ lysis buffer (TES, $10 \mathrm{mM}$ Tris/ $\mathrm{HCl}$, pH 7.5; $10 \mathrm{mM}$ EDTA; 0.5\% SDS). An equal volume of acid phenol was added and cells were incubated at $65^{\circ} \mathrm{C}$ for $60 \mathrm{~min}$ with occasional brief vortexing. Samples were then cooled on ice and spun in the microfuge. Acid/phenol extraction was repeated, followed by a chloroform extraction, keeping the samples on ice and centrifugation at $4{ }^{\circ} \mathrm{C}$. Sodium acetate was added $(3 \mathrm{M}, \mathrm{pH} \mathrm{5.3,40 \mu l)}$ and RNA was precipitated with $1 \mathrm{ml}$ ice-cold $100 \%$ ethanol. The RNA pellet was washed in $70 \%$ ice-cold ethanol and resuspended in $25 \mu \mathrm{H}_{2} \mathrm{O}$.

Separation of RNA was performed as described by Varela $e t$ al. (1995), DNA probes were labelled using the randompriming method (Prime-a-Gene labelling system; Promega). Signal quantification was performed by phosphoimaging (Phosphorimager 425; Molecular Dynamics). The PGK1 
Table 1. S. cerevisiae strains used

\begin{tabular}{|c|c|}
\hline Strain & Genotype \\
\hline EG123 & MAT $\alpha$ leu2-3 his 4 trp1 ura3-52 can1 \\
\hline W303IA & MAT $\alpha$ leu2-3/112 ura3-1 trp1-1 his3-11/15 ade2-1 can 1-100 GAL SUC malO \\
\hline$\Delta m s n 2 / 4$ & MAT $\alpha$ leu2-3/112 ura3-1 trp1-1 bis3-11/15 ade2-1 can 1-100 GAL SUC mal0 msn2:: HIS3 msn4:: TRP1 \\
\hline YPH 102 & MAT $\alpha$ ura 3 leu 2 his 3 ade 2 lys 2 \\
\hline JBY13 & MAT $\alpha$ ura 3 leu 2 his 3 ade 2 lys 2 hog $1::$ TRP1 \\
\hline SPI & MAT $\alpha$ leu 2 bis 3 trp1 ade 8 can1 ura3 \\
\hline S13-3A & MAT $\alpha$ leu2 bis 3 trp1 ade8 can1 ura3 tpk2::HIS3 tpk3::TRP1 bcy::LEU2 \\
\hline S18-D & MATa leu2 bis 3 trp1 ade8 can1 ura3 tpk1 w1 tpk2::HIS3 tpk3::TRP1 \\
\hline RS13-58A-1 & MAT $\alpha$ leu 2 bis 3 trp1 ade8 can1 ura3 tpk1w1 tpk2::HIS3 tpk3::TRP1 bcy::LEU2 \\
\hline
\end{tabular}

mRNA level was used as a loading control for cells grown on glucose. Because PGK1 mRNA levels decreased after high-salt challenge of cells grown on non-fermentable carbon sources, the $25 S$ rRNA level was used as additional loading control.

Western blotting and antibody staining. Tyrosine phosphorylation of Hog1p was examined in the EG123 strain carrying a HOG1 overexpression plasmid (pFJ0). Total protein samples were isolated as described by Davenport $e t$ al. (1995). Proteins were separated on $12.5 \%$ polyacrylamide gels and blotted onto nitrocellulose. The phosphotyrosine content of Hog1p was examined using the anti-phosphotyrosine monoclonal antibody PY20 (Transduction Laboratories). Anti-Hog1p antibody was kindly provided by Dr M. Gustin (Rice University, Houston, USA). Antibody binding was visualized using enhanced chemiluminescence (ECL; Amersham) after binding of a horseradish peroxidase-conjugated second antibody.

\section{RESULTS}

\section{PKA activity and the HOG pathway regulate the kinetics of HSP12 expression after high-osmolarity stress}

We have previously demonstrated that the HSP12 gene transcription is activated by the HOG pathway and repressed under conditions of high PKA activity (Varela et al., 1995). To discriminate between derepression and induction of the HSP12 expression, we were interested to know how the activity of PKA and HOG signalling are integrated in the regulation of HSP12 promoter activity. For this purpose we analysed the kinetics of induction of HSP12 mRNA levels after subjecting cells to high-osmolarity stress $(0.7 \mathrm{M} \mathrm{NaCl})$ in high- and lowPKA mutants as well as in the $\Delta h o g 1$ strain. As shown in Fig. 1(a) and quantified in Fig. 1(b), HSP12 mRNA levels reached a maximum $90 \mathrm{~min}$ after the addition of salt in the wild-type. When comparing the response in the PKA mutants, a shift of the maximum HSP12 mRNA levels to an earlier time-point is evident. From the analysis of mutant strains with either one of the Tpkp proteins, it is clear that all three TPK gene products can be involved in the repression of HSP12 gene expression (M. Siderius, unpublished). Therefore, there is a good correlation between the potential PKA activity (wild-type $>T P K 1$, $\left.t p k 2,3>t p k 1^{\mathrm{w}}, t p k 2,3\right)$ and the earlier timing of maximal HSP12 mRNA levels after salt stress. Furthermore, although at low levels, the high-PKA mutant $(t p k 2$, $t p k 3, b c y 1)$ still responded to high osmolarity with an increase in HSP12 mRNA level, as quantified in Fig. 1(c). The absolute levels of HSP12 mRNA were only affected significantly in the high-PKA strain. To analyse the input of the HOG pathway, high-osmolarity-induced HSP12 mRNA levels were examined in the $\Delta$ hog1 strain. The maximum HSP12 mRNA levels in response to high salt in this mutant appeared at a later time-point as compared to the wild-type (Fig. 2). Throughout this paper, we followed the assumption that the changes in mRNA levels are mainly a reflection of transcriptional regulation. It can not be excluded however that posttranscriptional events (e.g. mRNA stability) contribute to the observed effects.

\section{Carbon sources affect the output of stress-induced signalling}

To further analyse the contribution of presumed changes in PKA activity in the high-salt-induced derepression of HSP12 gene expression, we examined the response to $0.7 \mathrm{M} \mathrm{NaCl}$ of cells grown on glucose, ethanol or glycerol. The observation that HSP12 gene expression was derepressed in cells growing on non-fermentable carbon sources under non-stress conditions (Fig. $3 ; t=$ 0 ) might, at least partly, reflect a lower PKA activity as compared to glucose-grown cells. This assumption is supported by the carbon-source-mediated regulation of enzymes that were shown to be PKA targets, e.g. the glycolytic enzyme Pfk26p which is inactive in cells growing on ethanol and is activated in a PKA-dependent way when shifting to glucose as carbon source (François et al., 1984; Boles et al., 1996). Alternatively, loss of glucose repression of the HSP12 gene expression could also explain part of the derepression on non-fermentable carbon sources. In addition to the analysis of mutant strains, examining salt-induced HSP12 mRNA levels on non-fermentable carbon sources may give complementary information on the role of PKA in the stress response of yeast.

When yeast cells grown on non-fermentable carbon sources were exposed to high-osmolarity stress, growth arrest occurred comparable to the response on glucosebased medium (results not shown). This growth arrest 
(a) Wild-type

Time (min) ... $0 \quad 15 \quad 30 \quad 45 \quad 60 \quad 90 \quad 120$

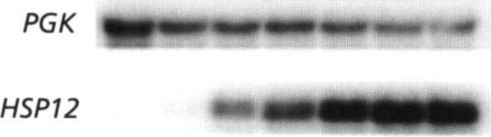

tpk2, tpk3

PGK

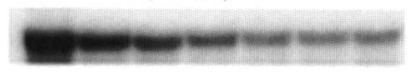

HSP12

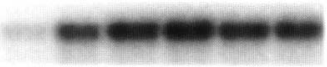

$t p k 1^{\mathrm{W}}, t p k 2, t p k 3$

PGK

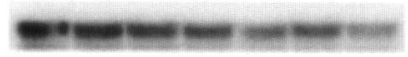

HSP12

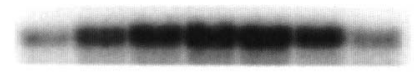

tpk2, tpk3, bcy1

PGK

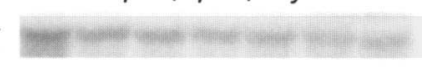

HSP12
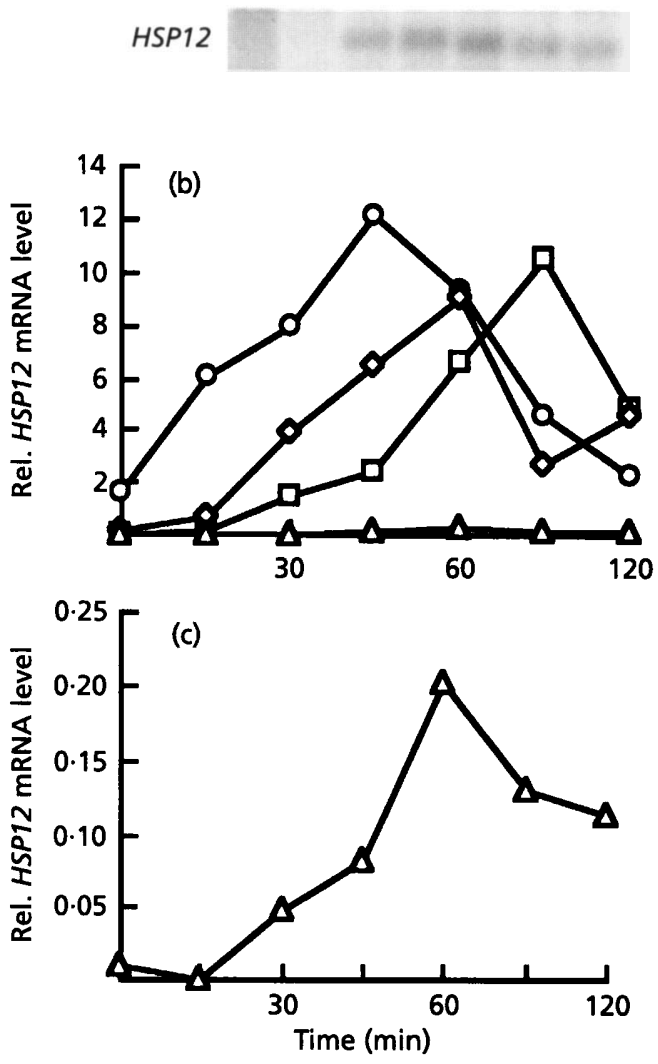

Fig. 1. High-salt-induced increase of HSP12 MRNA in PKA mutants. (a) Northern analysis of high-salt $(0.7 \mathrm{M} \mathrm{NaCl})$-induced mRNA levels of HSP12 and PGK1. (b) Quantification of HSP12 mRNA level relative to the 25S rRNA in the wild-type ( $\square$ ); tpk2, tpk3 ( $\diamond) ; t p k 1^{\mathrm{w}}$, tpk2, tpk3 (O) and tpk2, tpk3, bcy1 ( $\left.\triangle\right)$. (c) Quantification of HSP12 mRNA level relative to the $25 S$ rRNA in the tpk2, tpk3, bcy1 strain.

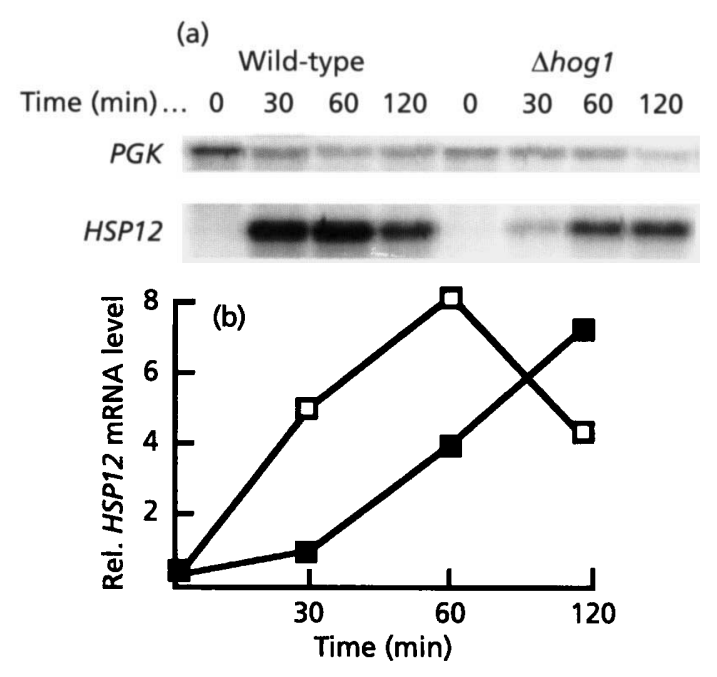

Fig. 2. High-salt-induced increase of HSP12 mRNA level in $\Delta$ hog1 strain. (a) Northern analysis of high-salt $(0.7 \mathrm{M} \mathrm{NaCl})$ -induced mRNA levels of HSP12 and PGK1. (b) HSP12 mRNA levels relative to PGK1 mRNA levels in the wild-type ( $\square)$ and $\Delta$ hog1 strain ( $\square$ ).

was reflected by the decrease of mRNA levels for ribosomal protein $\mathrm{S33}$, which accompanied the response to high-osmolarity stress (Fig. 3a). In contrast to the fairly constitutive presence of $P G K 1 \mathrm{mRNA}$ on glucosebased medium, high-osmolarity conditions on ethanolor glycerol-based media led to a marked decrease of PGK1 mRNA level. After exposure to $0.7 \mathrm{M} \mathrm{NaCl}$, induction of HSP12 gene expression varied greatly in cells grown on glucose-, ethanol- or glycerol-based media. Comparing the induction levels and kinetics, the induced HSP12 mRNA levels are lower on ethanolbased medium as compared to glucose (Fig. 3a, b). The lower level of induction is combined with a delay of the response in a W303IA background, but not in SPI (Fig. $3 c, d)$. When glycerol was used as carbon source, the non-stress level of HSP12 mRNA was found to be higher as compared with that on ethanol-based medium. Furthermore, when we analysed the high-salt-induced expression of HSP12 on glycerol-based medium, hardly any increase in mRNA level was observed. Apparently, HSP12 mRNA levels are influenced by factors that are dependent on the type of carbon source.

\section{Signalling through the HOG pathway after high- osmolarity challenge is diminished in glycerol-grown cells}

Activation of the HOG MAP kinase pathway after a high-osmolarity stress challenge results in the production of glycerol as osmolyte to compensate for the loss of water (Hohmann, 1997). The strong reduction of highsalt-induced HSP12 mRNA levels in cells that were grown with glycerol as sole carbon source could be a reflection of negative feedback on the HOG pathway activity by glycerol or one of its derivatives. To examine 
(a)
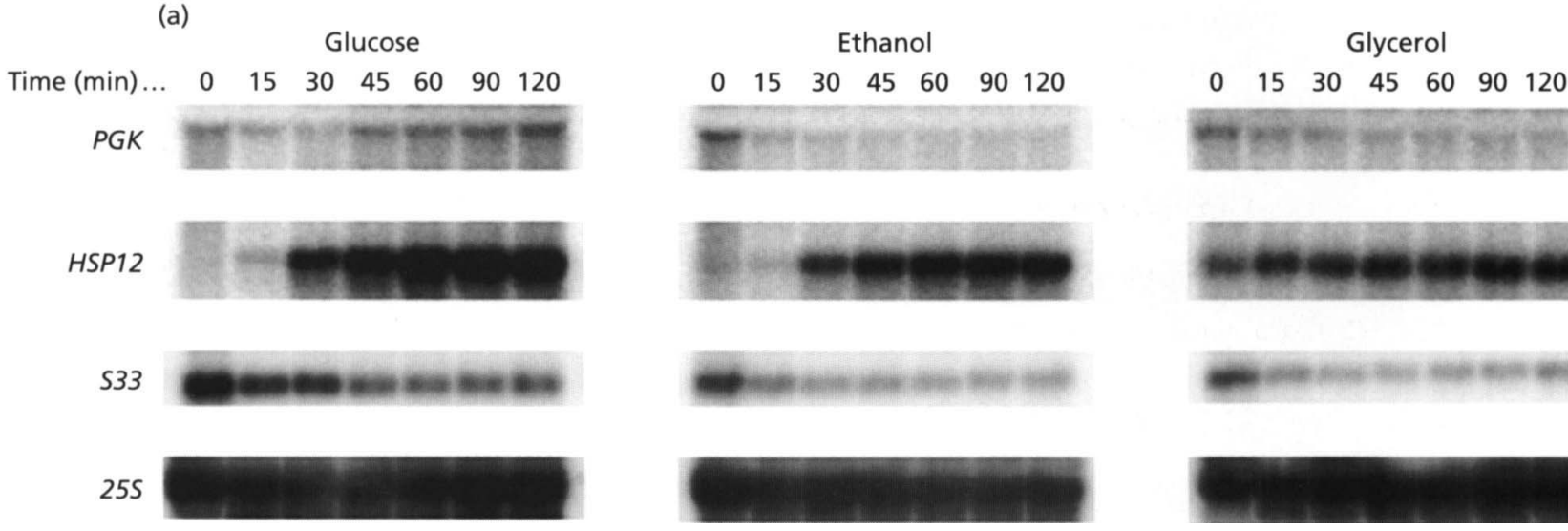

(b)
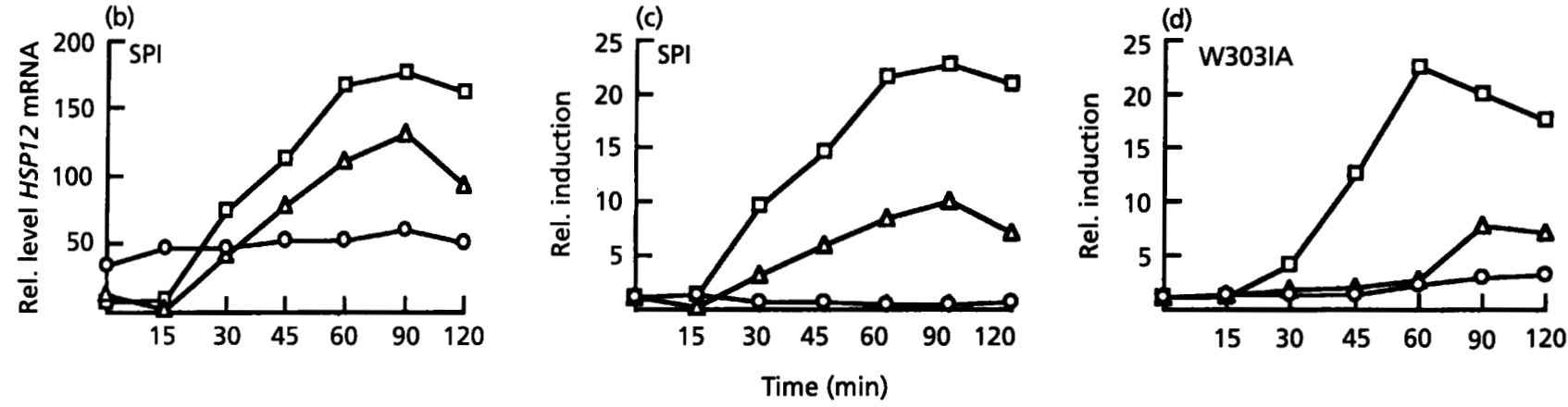

Fig. 3. High-salt-induced increase of HSP12 mRNA leveis on glucose-, ethanol- or glycerol-based media. (a) Northern analysis of mRNA levels after high-salt $(0.7 \mathrm{M} \mathrm{NaCl})$ exposure of SPI cells grown with either glucose, ethanol or glycerol as carbon source. (b) Quantification of relative (to 25S rRNA) level of HSP12 mRNA in strain SPI on glucose ( $\square$ ), ethanol ( $\triangle$ ) or glycerol (O). (c, d) Induction of HSP12 mRNA levels relative to the $t=0$ level in strains SPI (c) and W303IA (d).
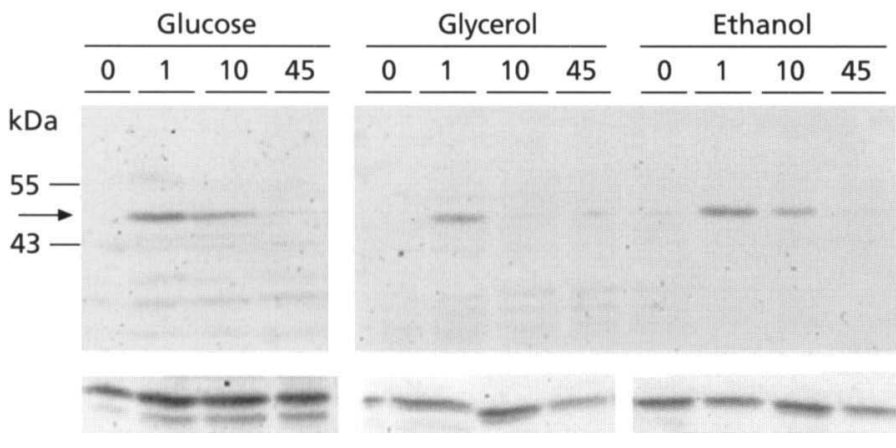

... Time $(\min )$

the effect of the different carbon sources on the activation of signal transduction through the HOG pathway, we analysed tyrosine phosphorylation of Hog1p by Western analysis in total cell extracts. We used a HOG1 overexpression strain to optimize the antiphosphotyrosine labelling without affecting the phosphorylation kinetics (Brewster et al., 1993). Hog1p is transiently phosphorylated on tyrosine after high-salt challenge, with a maximal phosphotyrosine level after $1 \mathrm{~min}$ (Fig. 4). When glycerol was used as carbon source, the response was more transient when compared with the response of glucose-grown cells (no signal at $t=$ $10 \mathrm{~min}$ ) and at a slightly lower level. Decreased salt-

$\alpha-\operatorname{Hog} 1 p$
Fig. 4. Western analysis of tyrosine phosphorylation of Hog1p after high-salt challenge. Total cell extracts $(40 \mu \mathrm{g})$ of yeast exposed to $0.7 \mathrm{M} \mathrm{NaCl}$ for times indicated on glucose-, ethanol- or glycerol-based media. Proteins were separated by SDSPAGE and blotted onto nitrocellulose. Phosphotyrosine content was detected with an anti-phosphotyrosine monoclonal antibody, PY20 $(1: 1000)$. The arrow indicates the position of Hog1p. Presence of Hog1p was detected with an anti-Hog1p polyclonal antibody. induced expression of the HSP12 gene on glycerol-based medium thus correlates with, and may at least be partly caused by, a decreased activation of Hog1p.

\section{Stress response on non-fermentable carbon sources is modulated by PKA activity}

The effect of glycerol on signalling through the HOG pathway did not explain the difference between highsalt-induced expression of HSP12 on ethanol-based media and that on glucose. Non-fermentable carbon source conditions probably also affect the stress response in another way. To investigate the effect of PKA activity 
(a) Wild-type

Time (min) .. $0 \quad 0 \quad 15 \quad 30 \quad 45 \quad 60 \quad 90 \quad 120$

PGK

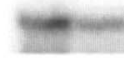

HSP12

$25 S$

(b)

Wild-type

Time (min) .. $0 \quad 15 \quad 30 \quad 45 \quad 60 \quad 90 \quad 120$

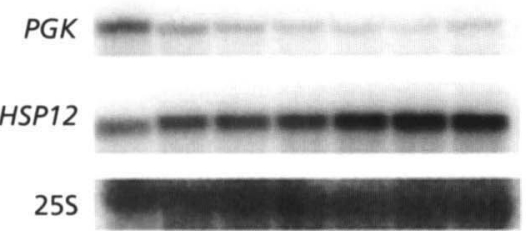

tpk $1 \mathrm{~W}, t p k 2, t p k 3$

$\begin{array}{lllllll}0 & 15 & 30 & 45 & 60 & 90 & 120\end{array}$

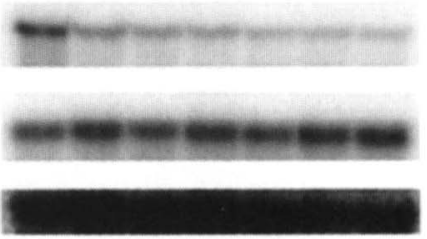

tpk $1 \mathrm{~W}$, tpk2, tpk3

$\begin{array}{lllllll}0 & 15 & 30 & 45 & 60 & 90 & 120\end{array}$

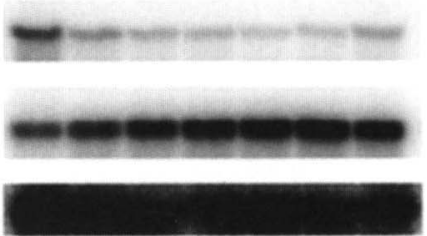

tpk2, tpk3, bcy1

$\begin{array}{lllllll}0 & 15 & 30 & 45 & 60 & 90 & 120\end{array}$

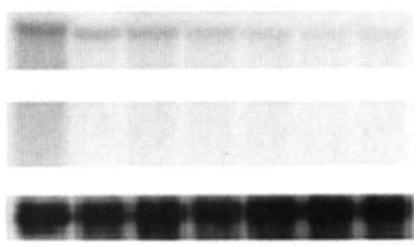

tpk2, tpk3, bcy1

$\begin{array}{lllllll}0 & 15 & 30 & 45 & 60 & 90 & 120\end{array}$

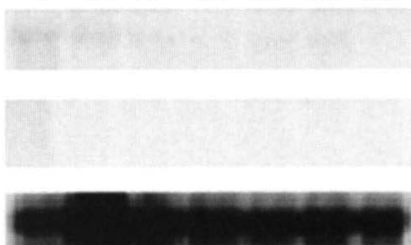

Fig. 5. High-salt-induced HSP12 mRNA levels in PKA mutants grown in ethanol- or glycerol-based media. (a) Northern analysis of HSP12 and PGK1 mRNA levels after high-salt $(0.7 \mathrm{M} \mathrm{NaCl})$ exposure of the low- (tpk 1 w, tpk2, tpk3) and high(tpk2, tpk3, bcy1) PKA strains grown on medium with ethanol as carbon source. (b) Northern analysis of high-salt-induced mRNA levels with the low- and high-PKA strains grown on glycerol-based medium.

on general stress signalling, we examined the HSP12 response on non-fermentable carbon sources in PKA mutants (Fig. 5). Using the low-PKA mutant $\left(t p k 1^{\mathrm{w}}\right.$, tpk2, tpk3), HSP12 mRNA levels were higher on nonfermentable carbon sources under control $(t=0)$ as well as stress-induced conditions. This indicated that derepression of HSP12 gene expression on ethanol- or glycerol-based growth media is incomplete. The additional induction of HSP12 mRNA levels after high-salt shock was diminished in the low-PKA mutant on ethanol-based medium comparable to the kinetics on glycerol-based medium.

The most dramatic effects on HSP12 gene expression were observed in the high-PKA $(t p k 2, t p k 3, b c y 1)$ strain: under both normal growth and stress conditions, HSP12 mRNA could not be detected. Catalytic activity of only Tpk1p (in the absence of the regulatory subunit Bcy1p) thus sufficed to overrule all responses on non-fermentable carbon sources. In contrast to the experiments performed with cells cultured in glucose-containing medium, no residual induction of HSP12 mRNA levels was apparent.

\section{PKA regulation of $H S P 12$ gene expression is mediated by Msn2/4p}

PKA activity (Marchler et al., 1993; Varela et al., 1995) and the STRE-binding proteins Msn2/4p have been implicated in signal transduction underlying the general stress response of $S$. cerevisiae (Martinez-Pastor et al., 1996). Deletion of the MSN2 and MSN4 genes results in decreased resistance of $S$. cerevisiae to a variety of severe stress conditions as well as loss of expression of general stress responsive genes (Martinez-Pastor et al., 1996). (a) Wild-type $\Delta m s n 2 / 4$
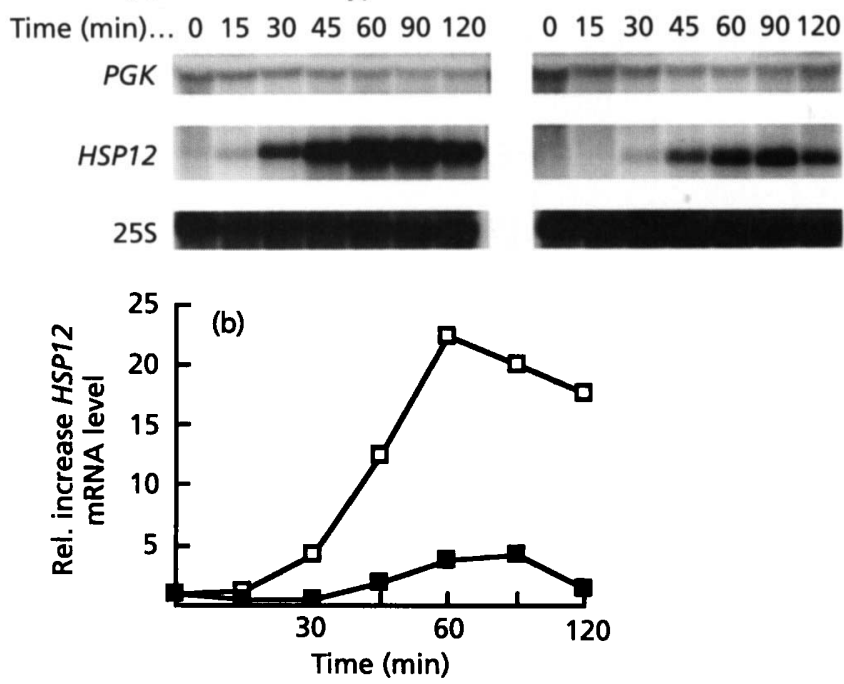

Fig. 6. High-salt-induced HSP12 mRNA levels in the $\triangle m s n 2 / 4$ strain. (a) Northern analysis of HSP12 and PGK1 mRNA levels after high-salt $(0.7 \mathrm{M} \mathrm{NaCl})$ exposure of the $\Delta m s n 2 / 4$ strain grown on glucose-based medium. (b) Quantification of relative (to 255 rRNA) level of HSP12 mRNA in the wild-type ( $\square$ ) and $\Delta m s n 2 / 4$ strain ( $\square$ ).

However, high-salt-induced increase in HSP12 mRNA levels is not completely abolished in the $m s n 2 / 4$ deletion strain (Fig. 6). A portion of the high-salt-induced increase in HSP12 mRNA levels might be derived from either residual Msn2/4p-independent input of the HOG pathway or another high-osmolarity-induced signal. Additional deletion of the PBS2 gene in the $\Delta m s n 2 / 4$ 
(b)
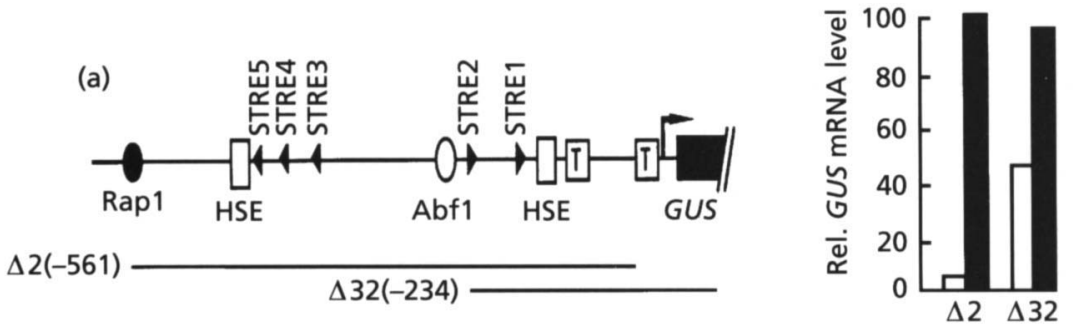

$\Delta m s n 2 / 4$

(c)

tpk1, tpk3, bcy1

Time $(\min ) \ldots 0 \quad 1530 \quad 566090120$

$\triangle 32-$ GUS

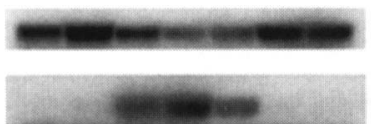

$\Delta 2-G U S$

HSP12

\section{$\begin{array}{lllllll}0 & 15 & 30 & 45 & 60 & 90 & 120\end{array}$}

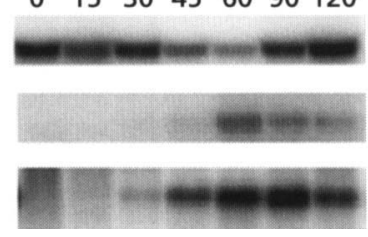

Fig. 7. High-salt-induced HSP12 promoter-GUS fusions in high-PKA mutant and $\Delta m s n 2 / 4$ strains. (a) Schematic representation of the $\Delta 2-$ and $\Delta 32-G U S$ constructs. Putative Abf1- and Rap1-binding sites in the HSP12 promoter are depicted, TATA boxes are shown as boxed Ts, heatshock elements are denoted by HSE and STRE sequences are depicted as arrowheads. (b) Relative (to the PGK1 mRNA level) GUS mRNA levels of the $\Delta 2$ - and $\Delta 32-G U S$ fusion constructs under non-stressed $(\square)$ and highsalt-induced ( $\square$ ) conditions. (c) Northern analysis of high-salt-induced $H S P 12, \Delta 2-$ and $\triangle 32-$ GUS mRNA levels in the high-PKA (tpk2, tpk3, bcy1) and $\Delta m s n 2 / 4$ strains. strain was previously shown to abolish the HSP12 response to $0.4 \mathrm{M} \mathrm{NaCl}$ (Martinez-Pastor et al., 1996). Although a direct link between PKA activity and the Msn2/4p has not yet been reported, stress-induced transport of the Msn2p to the nucleus appears to be correlated with PKA activity (H. Ruis, personal communication). By analysing different HSP12 promoterGUS fusion constructs in both the high-PKA mutant and $\Delta m s n 2 / 4$ strain we found further indications that Msn2/4p are likely to mediate the PKA effect on HSP12 promoter activity. In the promoter deletion studies that were reported by Varela et al. (1995), the proximal 561 bp fragment $(\Delta 2)$ of the HSP12 promoter was shown to contain all elements necessary for full function (Fig. $7 \mathrm{a}, \mathrm{b})$. Deletion of the $5^{\prime}$ part of the promoter up to the -234 position $(\Delta 32)$ resulted in a truncated promoter (still containing two STREs) that was constitutively derepressed and slightly induced by high-osmolarity stress (Fig. 7a, b). Because derepression of $\Delta 32$ could be due to loss of a promoter element regulated by PKA, we analysed regulation of the $\Delta 32-G U S$ constructs in the high-PKA strain as well as in the $\Delta m s n 2 / 4$ strain (Fig. $7 \mathrm{c})$. The $\Delta 2-$ GUS promoter construct was included in these analyses as the control for the full promoter activity. The $\triangle 2-G U S$ construct generally was regulated comparably to and following the kinetics of the endogenous HSP12 gene, although high-salt induction resulted in a somewhat more transient response, perhaps reflecting the instability of GUS mRNA (Fig. 7c). In contrast, the $\triangle 32-G U S$ construct was regulated in a remarkable fashion. In the high-PKA strain, exposure to $0.7 \mathrm{M} \mathrm{NaCl}$ resulted in an initial increase in $\triangle 32-$ GUS mRNA level, followed by a decrease of mRNA level inversely parallel to the increased levels of $\triangle 2-G U S$ mRNA and that of the endogenous HSP12 gene. Analysing the $\Delta 32-G U S$ construct in the $\Delta m s n 2 / 4$ strain showed the same kinetics. The observed kinetics of the $\triangle 32-$ GUS mRNA level was only observed in these strains upon high-osmolarity shock and not in a response to e.g. heat shock (not shown). These results (a) Wild-type

Time (min)... $0 \quad 15 \quad 3045 \quad 6090120$

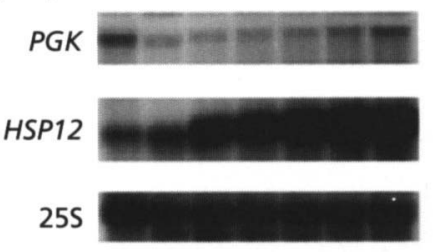

(b)

Time (min)... 01530456090120

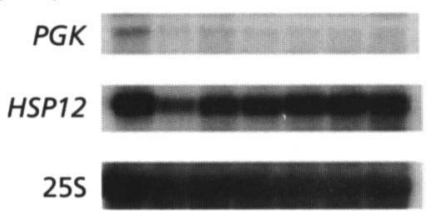

$\Delta m s n 2 / 4$

01530456090120

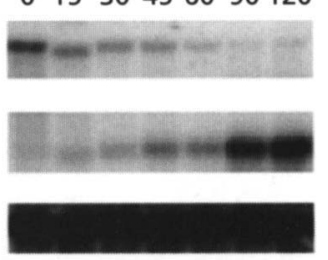

01530456090120

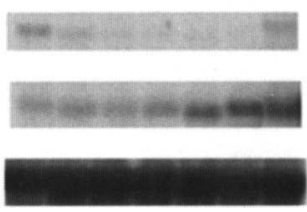

Fig. 8. High-salt-induced HSP12 mRNA levels in the $\triangle m s n 2 / 4$ strain grown in ethanol- or glycerol-based media. (a) Northern analysis of HSP12 and PGK1 mRNA levels after high-salt ( $0.7 \mathrm{M}$ $\mathrm{NaCl})$ exposure of the $\Delta m s n 2 / 4$ strain grown on medium with ethanol as carbon source. (b) Northern analysis of high-saltinduced mRNA levels in the $\triangle m s n 2 / 4$ strain grown on glycerolbased medium.

further suggest that the Msn2/4p and PKA function in a common signalling pathway, regulating promoter activity of the HSP12 gene.

\section{PKA activity regulates the stress response at least at two different levels}

To analyse at which level the non-fermentable carbon sources affected PKA input in the regulation of HSP12 gene expression in high-osmolarity conditions, we examined HSP12 expression after exposure of cells to $0.7 \mathrm{M} \mathrm{NaCl}$ in $\Delta m s n 2 / 4$ strain grown on ethanol- or glycerol-based media. If the influence of carbon sources 
on the regulation of stress-induced HSP12 gene expression would be mediated by a change in PKA activity, we would expect that $H S P 12$ expression in the $\Delta m s n 2 / 4$ strain is comparable to that in the high-PKA strain. In contrast to the high-PKA strain however, the HSP12 gene appeared to be still responsive to high-osmolarity shock in the $\Delta m s n 2 / 4$ strain on both ethanol- and glycerol-based media (Fig. 8a, b). Although diminished, HSP12 mRNA levels apparently follow the pattern of the induction in wild-type cells. However, the noninduced levels $(t=0)$ of HSP12 mRNA were strongly diminished when compared to wild-type on ethanol or glycerol. Therefore, Msn2/4p appears to be involved in the derepression of HSP12 gene expression when yeast is growing under non-fermenting conditions.

\section{DISCUSSION}

In previous studies on the regulation of high-osmolarityinduced HSP12 gene expression, the HOG pathway and PKA activity were shown to be involved in an opposite manner: the first by inducing expression, the latter by a stress-induced derepression. Here we compared the input of both pathways into the net induction of HSP12 expression after a high-salt challenge. We noticed that not only absolute mRNA levels, but also timing of the response is affected differently in mutants of both pathways. The shift of the high-salt-induced HSP12 response to a later time-point in the $\Delta$ hog 1 strain is indicative of involvement of this pathway in the early reponses to high osmolarity. Indeed, after a highosmolarity shock the phosphotyrosine level of Hog1p displays a rapid increase. Although the high-osmolarityinduced activation of the HOG pathway is rapid, the increase in mRNA level of responsive genes such as HSP12 and GPD1 (Albertyn et al., 1994; Varela et al., 1992) occurs relatively late. In the case of the HSP12 gene this could be a reflection of the repression of expression by PKA. Indeed, in low-PKA mutants a shift of the stress-induced peak in HSP12 mRNA levels to an earlier point in time was observed. In the $\Delta$ hog1 strain, still an increase in HSP12 mRNA levels was observed, indicative of stress-induced loss of PKA-mediated repression. Reduction of PKA activity as a response to stress might be involved in the later, adaptive, responses of $S$. cerevisiae to stress. Although there are reports of a high-salt-induced decrease of cAMP levels (Marquez $\&$ Serrano, 1996) and modulation of PKA activity by PBS2 (Boguslawski, 1992), the mechanism by which PKA activity may be lowered under high-osmolarity stress conditions is so far not understood. The intricate interplay of both PKA and HOG pathways makes it very hard to draw conclusions from the response to stress in signal transduction mutants analysed at a fixed point in time. Therefore, to analyse the response to high osmolarity under conditions in which PKA activity is expected to be lower, we chose to study this response in cells grown with ethanol or glycerol as carbon source instead of glucose. The derepression of HSP12 gene expression on non-fermentable carbon sources under non-stress conditions might be a reflection of lowered PKA activity.
The input of PKA activity in the regulation of HSP12 expression was also influenced on ethanol- and glycerolbased media as compared to medium containing glucose. The non-fermentable carbon sources gave rise to increased levels of HSP12 mRNA under non-stressed conditions. Since the derepressed levels of HSP12 mRNA were lower in the $\Delta m s n 2 / 4$ strain, derepression is probably regulated via lowered PKA activity acting through STRE-driven transcription. Derepression of the HSP12 gene expression was not complete because in the low-PKA mutant $\left(t p k 1^{\mathrm{w}}, t p k 2, t p k 3\right), H S P 12 \mathrm{mRNA}$ levels on ethanol or glycerol were even higher. Although derepression of the HSP12 gene expression on nonfermentable carbon sources was evident, the mRNA levels could still be induced after a high-salt challenge, even in a $\Delta$ hog1 strain (M. Siderius, data not shown). Therefore, we conclude that on both fermentable and non-fermentable carbon sources, salt-induced HSP12 mRNA expression may be determined in part by a decrease in PKA activity.

The molecular mechanism responsible for the previously observed derepression of the $\Delta 32-G U S$ construct (Varela et al., 1995) is elusive. Obviously the presence of two STREs is not sufficient for maintenance of the repressed state. In addition, transcription was found to be increased twofold upon salt challenge (Varela et al., 1995). Remarkably enough, opposite regulation was observed in the high-PKA and $\Delta m s n 2 / 4$ strains exposed to salt. We have no explanation for this intriguing finding. The comparable kinetics of the $\triangle 32-G U S$ expression in the high-PKA and $\Delta m s n 2 / 4$ strains, as shown in Fig. $7(\mathrm{c})$, however strongly suggest that signalling through Msn $2 / 4 p$ depends on regulation of PKA activity. On the other hand, if PKA input into the HSP12 expression would only be regulated by affecting STRE-driven promoter activity, the stress-induced HSP12 expression should be similar in the high-PKA and $\Delta m s n 2 / 4$ strains. The $\Delta m s n 2 / 4$ strain still showed a residual high-osmolarity response of $H S P 12$ on both ethanol- and glycerol-based media. Intriguingly, the high-PKA strain $(t p k 2, t p k 3, b c y 1)$ still showed a highosmolarity-induced increase in HSP12 mRNA level on glucose-based medium but no response at all on the nonfermentable carbon sources. Apparently, high PKA activity results in more severe effects on stress-induced expression than deletion of Msn2/4p, which is indicative of additional input of PKA activity in the regulation of this response.

Because the residual high-osmolarity-induced expression of the HSP12 gene in the $\Delta m s n 2 / 4$ strain is no longer observed when PBS2 is also deleted (MartinezPastor et al., 1996), it is likely that this induction is the net input of HOG pathway activation. The residual levels of high-salt-induced HSP12 mRNA observed in the high-PKA strain on glucose are probably also due to input of the HOG pathway. The complete absence of HSP12 mRNA on ethanol- or glycerol-based media in the high-PKA strain might be due to inhibition of the output of Hog1p activation. Although a minor decrease in tyrosine phosphorylation of Hog1p was observed on 
glycerol-based medium, high-salt-induced tyrosine phosphorylation was still detectable on both ethanol and glycerol. Therefore the level of inhibition of the output of the HOG pathway signalling most likely lies downstream of the MAP kinase.

Since the HOG pathway is very closely linked to the production of glycerol (Brewster et al., 1993), we tested whether the absence of a significant induction of HSP12 mRNA level in glycerol-grown cells could be due to feedback inhibition of glycerol or one of its metabolites. In contrast with ethanol-grown cells, activation of the HOG pathway as measured by the phosphotyrosine level of Hog1p was found to be affected in the glycerolgrown cells. Reduced activation of the HOG pathway may occur for example because glycerol or glycerol-3-P is metabolized into lipid components of the plasma membrane, changing the environment of the putative high-osmolarity receptors Sho1p or $\operatorname{Sin} 1 \mathrm{p}$. The response to lowering of the osmolarity in the medium has been proposed to involve signalling derived from sensing membrane stretch (Kamada et al., 1995). Whether a comparable mechanism is involved in regulation of the high-osmolarity response needs to be addressed in future research. Alternatively, compounds such as glycerol-3-P were proposed to affect histidine kinase activity by acting as phosphate donors (Posas et al., 1996). Utilization of glycerol as sole carbon source would therefore need to result in changes in lipid composition of the plasma membrane or higher glycerol-3-P concentration in the cell. We tried to mimick the glycerol effects by culturing the yeast cells in medium containing glycerol combined with glucose or ethanol. However, no significant change in the resulting kinetics of HSP12 gene expression as compared to that observed on glycerol was found (not shown). As uptake and metabolism of glycerol could be different under these conditions, these experiments might not be conclusive.

\section{Concluding remarks}

To summarize the data presented in this paper, we observed that stress-induced reduction of PKA activity mediates HSP12 expression through $\mathrm{Msn} 2 / 4 \mathrm{p}$ and STRE-driven promoter activity but also by affecting an unknown component(s). Exposure to osmotic stress resulted in a residual increase in HSP12 in the high-PKA strain grown in the presence of glucose. On nonfermentable carbon sources, however, this residual response was lost. Because the $\Delta m s n 2 / 4$ strain is still responsive on ethanol or glycerol, absence of the HSP12 gene expression in the high-PKA strain is due to absence of input of PKA into the other part of the stress response. This regulation is mediated through a component which is regulated by glucose and might control the output of Hog1p activation. Since regulation of the main glucose repression pathway seems to fit into the above-mentioned characteristics in being regulated by PKA activity and being glucose-regulated, we hypothesize that part of the high-osmolarity induction of HSP12 gene expression might be influenced by components of this pathway. Connection of this signalling pathway with the stress
Working model of high-osmolarity-induced HSP12 gene expression

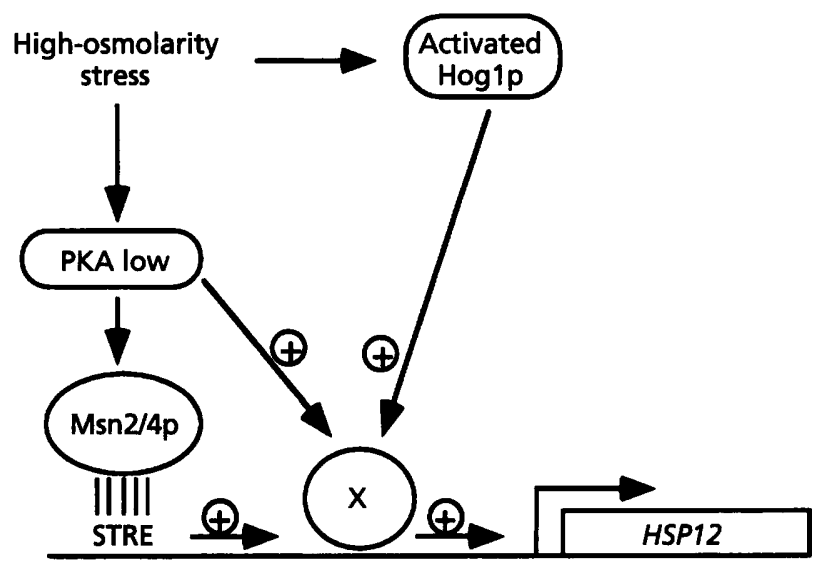

Fig. 9. Hypothetical model of the regulation of highosmolarity-induced HSP12 expression. Exposure of S. cerevisiae to high-osmolarity conditions leads to activation of HSP12 gene expression mediated by Hog1p activation and reduction of PKA activity. PKA regulation of the HSP12 promoter activity is mediated by Msn2/4p and another factor, $X$, which affects output of the HOG pathway on non-fermentable carbon sources. Considering the glucose- and PKA-mediated regulation and links of multicopy suppressors of $\Delta s n f 1$ with the stress response, component(s) of the main glucose repression pathway might be involved in this regulation. Factor $X$ might be a component of a stress-response pathway upstream of the transcription machinery or could be part of the mediator complex, regulating affinity or transcriptional activity of RNA polymerase II holoenzyme.

response is not unlikely as several multicopy suppressors of the $\Delta s n f 1$ strain (MSN2/4, ROX3) appear to influence stress-induced gene expression (Evangelista et al., 1996; Martinez-Pastor et al., 1996). Interaction between glucose-regulated and stress-response signalling could occur at the level of signal transduction components and/or by influencing transcriptional activity or affinity of the RNA polymerase II holoenzyme via components of the mediator complex (these options are encapsulated in ' factor X' in the working model presented in Fig. 9). We are currently testing this working hypothesis and preliminary experiments with mutants in the main glucose repression pathway indeed indicate involvement of this pathway with the high-osmolarity response of the HSP12 gene.

\section{ACKNOWLEDGEMENTS}

We thank Dr M. Gustin (Rice University, Houston, USA) for providing the anti-Hog1 antibody and Dr F. Estruch (Universitat de Valencia, Spain) and Dr J. Winderickx (Katholieke Universiteit, Leuven, Belgium) for providing yeast strains. This work was carried out as part of the EU 'glycerol project' (grant BIO4-CT950161, to M.S.).

\section{REFERENCES}

Albertyn, J., Hohmann, S., Thevelein, J. M. \& Prior, B. A. (1994). GPD1, which encodes glycerol-3-phosphate dehydrogenase, is essential for growth under osmotic stress in Saccharomyces 
cerevisiae, and its expression is regulated by the high-osmolarity glycerol response pathway. Mol Cell Biol 14, 4135-4144.

Boguslawski, G. (1992). PBS2, a yeast gene encoding a putative protein kinase, interacts with the Ras2 pathway and affects osmotic sensitivity of Saccharomyces cerevisiae. J Gen Microbiol 138, 2425-2432.

Boles, E., Gohlmann, H. W. H. \& Zimmermann, F. K. (1996). Cloning of a second gene encoding 6-phospho-2-kinase in yeast, and characterization of mutant strains without fructose-2,6bisphosphate. Mol Microbiol 20, 65-76.

Brewster, J., De Valoir, T., Dwyer, N. D., Winter, E. \& Gustin, M. C. (1993). An osmosensing signal transduction pathway in yeast. Science 259, 1760-1763.

Coote, P. J., Cole, M. B. \& Jones, M. V. (1991). Induction of increased thermotolerance in Saccharomyces cerevisiae may be triggered by a mechanism involving intracellular $\mathrm{pH}$. J Gen Microbiol 137, 1701-1708.

Davenport, K., Sohaskey, M., Kamada, Y., Levin, D. E. \& Gustin, M. C. (1995). A second osmosensing signal transduction pathway in yeast. Proc Natl Acad Sci USA 270, 30157-30161.

Evangelista, C. C., Rodriguez Torres, A. M., Limbach, M. P. \& Zitomer, R. S. (1996). Rox3 and RTS1 function in the global stress response pathway in bakers yeast. Genetics 142, 1083-1093.

François, J., Van Schaftingen, E. \& Hers, H. G. (1984). The mechanism by which glucose increases fructose 2,6-bisphosphate concentration in Saccharomyces cerevisiae. Eur J Biochem 145, 187-193.

Griffioen, G., Mager, W. H. \& Planta, R. J. (1994). Nutritional upshift response of ribosomal protein gene transcription in Saccharomyces cerevisiae. FEMS Microbiol Lett 123, 137-144.

Hohmann, S. (1997). Shaping up: the responses of yeast to osmotic stress. In Yeast Stress Responses, pp. 101-146. Edited by S. Hohmann \& W. H. Mager. Georgetown, TX: R. G. Landes.

Hohmann, S. \& Mager, W. H. (1997). Yeast Stress Responses. Molecular Biology Intelligence Unit. Georgetown, TX: R. G. Landes.

Kamada, Y., Jung, U. S., Piotrowski, J. \& Levin, D. E. (1995). The protein kinase C-activated MAP kinase pathway of Saccharomyces cerevisiae mediates a novel aspect to the heat shock response. Genes Dev 9, 1559-1571.

Kobayashi, N. \& McEntee, K. (1993). Identification of cis and trans components of a novel heat shock stress regulatory pathway in Saccharomyces cerevisiae. Mol Cell Biol 13, 248-256.

Larsson, K., Eriksson, P., Ansell, R. \& Adler, L. (1993). A gene encoding sn-glycerol-3-phosphate dehydrogenase $\left(\mathrm{NAD}^{+}\right)$complements an osmosensitive mutant of $S$. cerevisiae. Mol Microbiol 10, 1101-1110.
Levin, D. E. \& Errede, B. (1995). The proliferation of MAP kinase signalling pathways in yeast. Curr Opin Cell Biol 7, 197-202.

Maeda, T., Wurgler-Murphy, S. M. \& Saito, H. (1994). A twocomponent system that regulates an osmosensing MAP kinase cascade in yeast. Nature 369, 242-245.

Maeda, T., Takekawa, M. \& Saito, H. (1995). Activation of yeast PBS2 MAPKK by MAPKKKs or by binding of an SH3-containing osmosensor. Science 269, 554-558.

Marchler, G., Schuller, C., Adam, G. \& Ruis, H. (1993). A Saccharomyces cerevisiae UAS element controlled by protein kinase A activates transcription in response to a variety of stress conditions. EMBO J 12, 1997-2003.

Marquez, J. \& Serrano, R. (1996). Multiple transduction pathways regulate the sodium-extrusion gene $P M R 2 / E N A 1$ during salt stress in yeast. FEBS Lett 382, 89-92.

Martinez-Pastor, M. T., Marchler, G., Schuller, C., Marchler-Bauer, A., Ruis, H. \& Estruch, F. (1996). The Saccharomyces cerevisiae zinc finger proteins Msn2p and Msn4p are required for transcriptional induction through the stress-response element (STRE). EMBO J 15, 101-109.

Posas, F., Wurgler-Murphy, S. M, Maeda, T., Witten, E. A., Thai, T. C. \& Saito, H. (1996). Yeast HOG1 MAP kinase cascade is regulated by a multistep phosphorelay mechanism in the SNL1YPD1-SSK1 two-component osmosensor. Cell 88, 865-875.

Ruis, H. \& Schuller, C. (1995). Stress signalling in yeast. Bioessays $17,959-965$.

Schmitt, A. \& McEntee, K. (1996). Msn2p, a zinc finger DNAbinding protein, is the transcriptional activator of the multistress response in S. cerevisiae. Proc Natl Acad Sci USA 93, 5777-5782.

Siderius, M. \& Mager, W. H. (1997). General stress response: in search of a common denominator. In Yeast Stress Responses, pp. 213-230. Edited by S. Hohmann \& W. H. Mager. Georgetown, TX: R. G. Landes.

Thevelein, J. M. (1994). Signal transduction in yeast. Yeast 10, 1753-1790.

Trollmo, C., André, L., Blomberg, A. \& Adler, L. (1988). Physiological overlap between osmotolerance and thermotolerance in Saccharomyces cerevisiae. FEMS Microbiol Lett 56, 321-326.

Varela, J. C. S., van Beekvelt, C. A., Planta, R. J. \& Mager, W. H. (1992). Osmostress-induced changes in yeast gene expression. Mol Microbiol 6, 2183-2190.

Varela, J. C. S., Praekelt, U. M., Meacock, P. A., Planta, R. J. \& Mager, W. H. (1995). The Saccharomyces cerevisiae HSP12 gene is activated by the high-osmolarity glycerol pathway and negatively regulated by the protein kinase A. Mol Cell Biol 15, 6232-6245.

Received 16 April 1997; revised 17 June 1997; accepted 19 June 1997. 